\title{
Color Correction: A Novel Weighted Von Kries Model Based on Memory Colors
}

\author{
Alejandro Moreno*, Basura Fernando, Bismillah Kani, \\ Sajib Saha, and Sezer Karaoglu \\ Color in Informatics and Media Technology (CIMET) \\ \{alejandro.moreno, sezer.karaoglu, sajib.saha\}@hig.no, \\ \{bismillahkani, basuraf\}@gmail.com \\ http://www .master-erasmusmundus-color.eu/
}

\begin{abstract}
In this paper we present an automatic color correction framework based on memory colors. Memory colors for 3 different objects: grass, snow and sky are obtained using psychophysical experiments under different illumination levels and later modeled statistically. While supervised image segmentation method detects memory color objects, a luminance level predictor classifies images as dark, dim or bright. This information along with the best memory color model that fits to the data is used to do the color correction using a novel weighted Von Kries formula. Finally, a visual experiment is conducted to evaluate color corrected images. Experimental results suggest that the proposed weighted von Kries model is an appropriate color correction model for natural images.
\end{abstract}

Keywords: Color Correction, Memory Color, Von Kries Model, Multispectral Images.

\section{Introduction}

Memory colors can be understood as a phenomenon in which an object's characteristic color influences our perception of its color. According to Fairchild, memory colors can be considered a phenomenon where, in our minds, a particular object has a particular color associated to it. This happens even though the actual color is often different from what we remember [2. Research shows that memory colors are usually located in a compact area within the chromaticity space [11; having a narrow range of hue values, even for different people. This conveys that memory colors can be generalized, and although they can change depending on several factors related to culture [4] [12, the fact that people perceive certain objects irremediably associated to particular colors cannot be overlooked.

\footnotetext{
* This work is part of CIMET project contest organized by Nokia Corporation and CIMET. Special thanks to University of Eastern Finland and Gjovik University College, Norway.
} 
These characteristics of memory color objects have inspired new trends in research to analyze the cognitive effects and cultural dependencies of memory colors, the definition of new memory color categories and ways in which to apply this information in higher level image analysis and processing stages. In [3] memory color has been used for image segmentation and classification. Zhang and Quan use 3 memory color categories to determine different illuminants present in an image by clustering pixels based on memory color information [15]. In [10] Aitao Lu et al. show that memory colors play a key role in early perceptual processes for object recognition.

Kaiqi et al. [13] propose the use of object recognition for automatic color constancy, but their approach requires one or more of the training objects to be present in the analyzed image. The use of visual object categories was also considered in [14, but the estimation method they present is based solely on utilizing the mean color values of the categories without any further analysis in the chromaticity domain. Moreover the evaluation method introduced is rather expensive to compute.

One of the most recent and interesting works based on memory colors was done by Esa Rahtu et al. in [11, where data of memory color objects present in an image was used to shift the color values for automatic color correction. Since most of the work done in color correction is based on low-level image information, the use of high level information such as memory color objects and semantic content of images to perform such task proves to be novel and highly interesting. Nonetheless, it still presents some shortcomings: the model claims to provide visually pleasing images but no psychopshysical experiment was carried out to prove this. Also, the model works for only one memory color object present in each image, thus the presence of several memory color objects forces the model to choose one of them to perform the correction.

This paper solves these 2 shortcomings while addressing some new and interesting questions about memory colors. First, an analysis to find out the difference, if any, of memory colors under different lighting conditions was carried out. Two different experiments were performed, one where users were asked to recall memory colors without any visual aid and another were images were used to provide context information. Second, a novel color correction formula based on a weighted Von Kries model is proposed. The model works for any number of memory color objects present simultaneously in the image. Third, a pioneer approach to collect memory color data by using multispectral images in the psychophysical experiments is used. The paper is structured as follows: The proposed framework is described in section 2 ; section 3 explains memory color data acquisition process; section 4 describes the proposed color correction model; section 5 illustrates the experimental results and finally the conclusion in section 6 .

\section{Proposed Framework}

The proposed framework is two fold, (1) memory color data acquisition and modeling (2) color correction process. The overall process is represented in Fig. 1] and Fig. 2, 


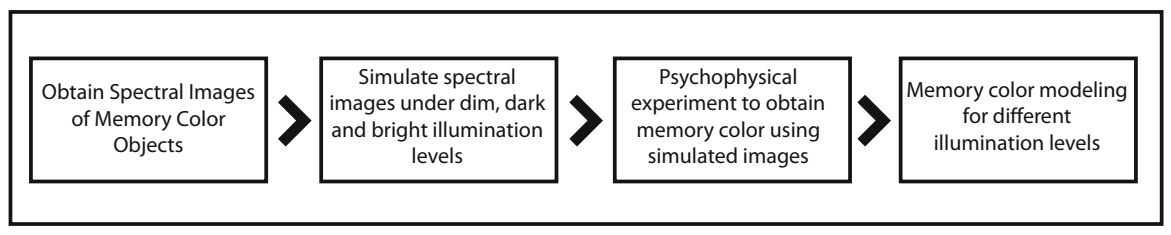

Fig. 1. Memory color data acquisition and modeling process

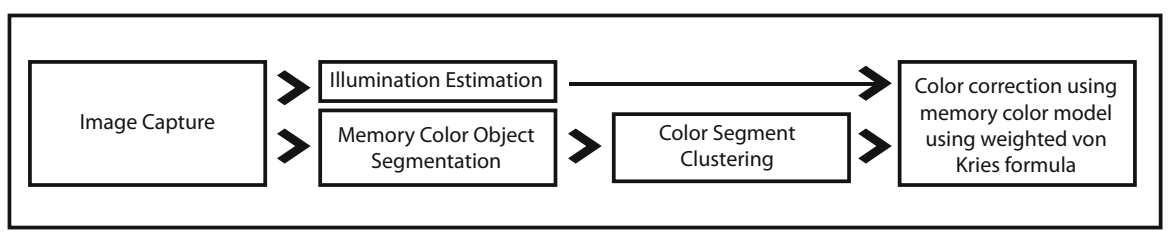

Fig. 2. Color correction process

First, memory color spectral images were captured and simulated for three different illumination levels: dark, dim and bright. Memory color objects were grayed out of the images and shown to 19 observers, whom were asked to vote for the most preferable memory color under a given illumination level for each object (memory colors obtained using images as visual aid will be called preferable memory colors). Afterwards, a second experiment was performed to obtain memory colors based solely on the recall of the subjects. The selected memory color patches were measured in CIE XYZ coordinates using a colorimeter considering the surround illumination. For each memory color object, reported colors were modeled using an ellipsoid in CIELab space for each illumination level.

The color correction process started by estimating the image illumination and segmenting the memory color objects. Knowing the illumination level, finding the most suitable model for a given memory color was possible. The image was segmented into several memory color regions and each memory color was partitioned into two clusters using K-means to reduce intra class variability and discarding outliers. After this, the weighted von Kries color correction model was applied based on the closest memory color for the selected model and the mean of the color cluster. In all the experiments and models sky, grass and snow were used as memory color objects however, the proposed framework and model can be extended to other memory color objects too.

\section{Memory Color Data}

\subsection{Multispectral Image Acquisition}

The advantage of using multi-spectral images is that it is possible to simulate the exact same image under known luminance levels and light sources. The spectral images were captured using the Nuance liquid crystal tunable filter (LCTF) 
spectral camera (Fig. 3) that works on visible range. The model is Nuance FX. Spectral data with the dynamic of 12 bits was acquired using optimal integration times for each measured channel with the imaging software (Nuance 2.4) and the data was further analyzed with a custom-made MATLAB program. CIEXYZ color coordinates were calculated using 1931 human observer color matching function and the illuminant chosen was 'D65'. The standard reference white was placed in the image area and acquired along with the image which was later used to correct the raw spectral reflectance data. Images were simulated for three luminance levels selected as reflective values of $20 \%, 50 \%$ and $100 \%$.

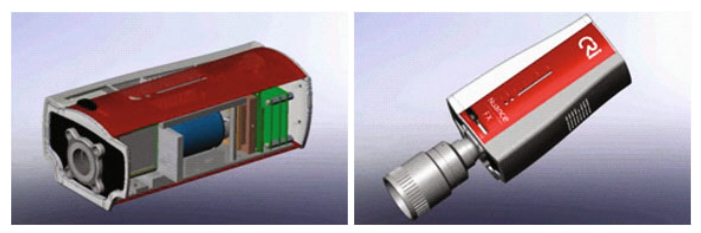

Fig. 3. Nuance liquid crystal tunable filter (LCTF) spectral camera

\subsection{Memory Color Experiment}

Nineteen observers volunteered for the memory color experiment: 14 males and 5 females; all with normal color vision, as confirmed by the Ishihara test plates screening method [6]. The mean age for all the observers was 24.6 (range 21 to 27). A matte white lighting cabinet with diffuse illumination was used to simulate each of the three different lighting condition levels: bright, dim and dark. Mean luminance levels of 2230 lux, 650 lux and 170 lux were used to simulate each lighting condition. The patches used for color selection were taken from the printed Munsell Chart.

Each observer participated in two short sessions. In the first session users were asked to recall the color of sky, grass and snow under a particular illumination and then select the Munsell patch that most closely resembled the color in his memory. Afterwards, the illumination level was changed and after the user had adapted, he was asked to repeat the procedure. This was done for the three luminance levels and the colors obtained through this method were called memory colors.

In the second session the procedure was the same but instead of recalling the color, the spectral images simulated under different illumination were displayed to the user. In these images, the memory color object was grayed out. The user was asked to select the color he would like to give to the memory color object using the Munsell patches once they had adapted to the different lighting conditions. These colors were called preferable memory colors.

In each session intra observer variability was taken into consideration by showing the color patches in random order 3 times for each observer. 


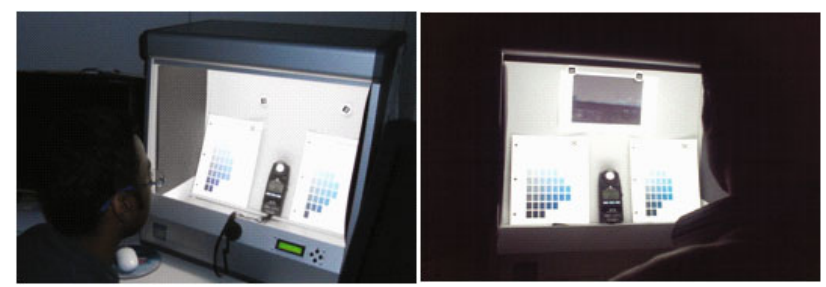

Fig. 4. Memory color visual experiments

\subsection{Memory Color Modeling}

The obtained memory color data was modeled as ellipses, for each lighting level and memory color object [11. Instead of representing the memory color in linear RGB space, the use of $\mathrm{L}^{*} \mathrm{a}^{*} \mathrm{~b}^{*}$ color space was proposed. $\mathrm{L}^{*} \mathrm{a}^{*} \mathrm{~b}^{*}$ color space is device independent and is developed for color difference measurements. Mean vector $m$ and covariance matrix $\mathrm{C}$ were calculated for each instance of memory color obtained from visual experiments. Afterwards, the memory color ellipses were modeled using the following equation.

$$
r_{m}^{2}=(x-m)^{T} C^{-1}(x-m) .
$$

Here $r_{m}$ is a parameter of the model. Given a data point $x, x$ will be included in the ellipse, if corresponding $r_{m}$ value is greater than some threshold.

\section{Color Correction Model}

\subsection{Memory Color Segmentation}

The main goal of memory color object detection and segmentation was to assist the color correction based on the memory color objects present in the image. To recognize memory color objects, a simple and fast supervised machine learning approach is proposed in this paper. 38 images which contain sky, grass and snow were collected; 18 of them were used for training and 20 for testing. First, patches that contain sky, grass and snow in the training images were manually labeled. Afterwards all images were transformed into $\mathrm{L}^{*} \mathrm{a}^{*} \mathrm{~b}^{*}$ opponent color space and for each pixel a 3D color histogram consisting of eight bins for each channel was computed for a local neighborhood of size 7x7. Afterwards the 512 bin histogram is transformed into 1-D histogram using concatenation. This local color descriptor was used as a feature vector. To reduce the dimensionality PCA was applied on all training samples until $90 \%$ of the energy remained in the signal. For training, Random Forest classifier [1] was used with 8 random features and 20 trees. 200000 samples were selected randomly to learn about grass, snow and sky. The test set was also labeled for grass, snow and sky to measure the classification accuracy. A $93 \%$ classification rate is reported using Random Forest [1] 
classifier. The Random Forest classifier is an ensemble classifier consists decision trees and outputs the class that is the mode of the class's output by individual trees. It is a very fast classifier compared to many other classification algorithms.

\subsection{Illumination Estimation}

The estimation of the lighting condition of an image still poses a series of difficulties. Even if an image is taken in broad daylight, if using only color analysis, dark color and shadows might lead to a misclassification or a bad estimation of the lighting condition. Currently there are algorithms that are able to obtain the lighting information accurately by applying complex and computationally expensive algorithms. In [9], not only the lighting condition of an image is obtained with a high accuracy but also the light sources and positions. Nonetheless, a representative object in the input image needs to be manually outlined to be able to perform the analysis. This and similar approaches while effective cannot be applied in the proposed system since low to mid power devices are targeted and their computation becomes prohibitive. Also, the input images can not undergo any kind of manual preprocessing.

Taking into consideration these restrictions, a more simple yet precise approach had to be considered. In [8] the authors propose a novel algorithm for image enhancement based on a multi-scale wavelet transform and a statistical approach to image representation proposed by Jobson in [7. The statistical approach provided a simple, fast and effective method to classify the images without modifying them. Jobson states that good visual representation of images converge to some statistical characteristics. Based on this fact, 25 randomly selected images from the internet were analyzed under 3 different lighting conditions, same as the multispectral data analyzed previously: bright, dim and dark. Working with the RGB values would be the most common practice, however this color space has some undesirable properties like correlation between color channels. Hence, the use of the iHLS color space [5] was preferred due to the separation of chromaticity information from lightness information, and its brightness channel independence from the saturation channel. The overall lightness was obtained by taking the mean of the lightness values of the whole image. Afterwards K-Means clustering was performed to obtain the values which were used to classify the input images. Three well defined clusters were obtained, one for each lighting condition as seen in Fig 5. An $89 \%$ classification accuracy is obtained for our test set.

\subsection{Color Correction}

Let us consider a set of memory color objects R. Color correction is done using weighted von Kries $[2$ model after the image has been segmented into $|R|$ number of memory color regions using supervised segmentation. Weights of each component are determined by two factors. First, the distance to the closest memory 


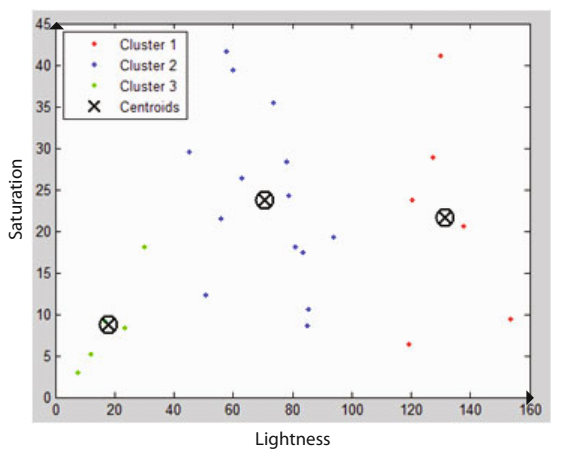

Fig. 5. Lightness clusters for the train images

color in the memory color model. Second, the normalized area of the memory color region in the segmented image. Each memory color segment in the image was represented by one representative $\mathrm{p}$ by taking the mean color of memory color segment, or multiple representatives by applying K-means algorithm.

Using the elliptic model $\mathrm{x}$ of the memory color object $r(r \in R)$ at the classified lighting level, and the memory color segment representative $\mathrm{p}$ in the image, the distance between them is calculated as follows:

$$
d_{r}=\min D(x, p) .
$$

where $\mathrm{D}$ is $\Delta E$ or the color difference formula in a given color space. The normalized area of a given memory color segment $r,(r \in R)$ is denoted by $A_{r}$ $\left(0 \leq A_{r} \leq 1\right)$. The weight factor for a given memory color segment $\mathrm{r}$ is defined as follows.

$$
w_{r}=\frac{A_{r}}{d_{r}} .
$$

The normalized weight factor $W_{r}$ is defined as follows.

$$
W_{r}=\frac{w_{r}}{\Sigma_{\forall i \in R} w_{i}}
$$

Suppose the $i^{\text {th }}$ memory color closest vector to the model is represented by $m_{i}$ and $m_{i}=\left[m_{i}^{r}, m_{i}^{g}, m_{i}^{b}\right]$ where rgb is the cone response color space. Suppose in the image the corresponding memory color object representative vector is given by $p_{i}=\left[p_{i}^{r}, p_{i}^{g}, p_{i}^{b}\right]$. The color correction for an input pixel having intensity $[\mathrm{R}, \mathrm{G}, \mathrm{B}]$ is corrected to $\left[R_{c}, G_{c}, B_{c}\right]$ using the following weighted von Kries formula.

$$
\begin{aligned}
& R_{c}=\left(\Sigma_{i} W_{i} \frac{p_{i}^{r}}{m_{i}^{r}}\right) \times R . \\
& G_{c}=\left(\Sigma_{i} W_{i} \frac{p_{i}^{g}}{m_{i}^{g}}\right) \times G .
\end{aligned}
$$




$$
B_{c}=\left(\Sigma_{i} W_{i} \frac{p_{i}^{b}}{m_{i}^{b}}\right) \times B
$$

The same color correction can be represented in matrix form as follows.

$$
\left|\begin{array}{l}
R_{c} \\
G_{c} \\
B_{c}
\end{array}\right|=\left|\begin{array}{ccc}
\frac{p_{i}^{r}}{m_{i}^{r}} & \frac{p_{i+1}^{r}}{m_{i+1}^{r}} & \frac{p_{i+2}^{r}}{m_{i+2}^{r}} \\
\frac{p_{i}^{g}}{m_{i}^{g}} & \frac{p_{i+1}^{g}}{m_{i+1}^{g}} & \frac{p_{i+2}^{g}}{m_{i+2}^{g}} \\
\frac{p_{i}^{b}}{m_{i}^{b}} & \frac{p_{i+1}^{b}}{m_{i+1}^{b}} & \frac{p_{i+2}^{b}}{m_{i+2}^{b}}
\end{array}\right| \otimes\left|\begin{array}{c}
W_{i} \\
W_{i+1} \\
W_{i+2}
\end{array}\right| \odot|R G B|^{T} .
$$

A better representation of the memory color segments in an image is achieved using multiple representatives through a clustering algorithm to reduce the intra class color variability. The proposed method uses K-means algorithm but it is also possible to use any density based clustering algorithm such as meanshift or mixture of Gaussians. The experimental results show that two clusters are sufficient to represent accurately memory color segments in an image and to successfully apply color correction. Applying color correction using 2 representatives for the 3 memory color categories result in 8 color corrected images. Visual experiments were carried out to find the best results. Experimental results suggest that memory color varies a lot from one observer to another. Color correction solely based on memory color data may not be the most suitable. Therefore preferable memory colors for grass, sky and snow were collected. These colors were also used in the color correction process using the same framework. In the next section a comparison study of these color correction methods is carried out.

\section{Experimental Results}

The summary of the experimental results obtained in visual experiments for memory color acquisition is shown in table 1 and table 2 isual experiment to analyze the performance of our algorithm was also carried out. Two color correction datasets were developed: one based on the memory color model and the other based on preferable color model. Each model generated nine color corrected images. Users were shown eight color corrected images along with the original image and were not aware of the presence of the original image. 235 observers participated in the experiment which consisted in choosing the most preferable or pleasing image. When preferable memory color model based correction was used, $23 \%$ of the users liked the original image while $77 \%$ preferred the color corrected image. On the other hand, $40 \%$ preferred the original image when memory color based correction was used. In overall, $30 \%$ of the users preferred the original image while $70 \%$ preferred the color corrected images. 


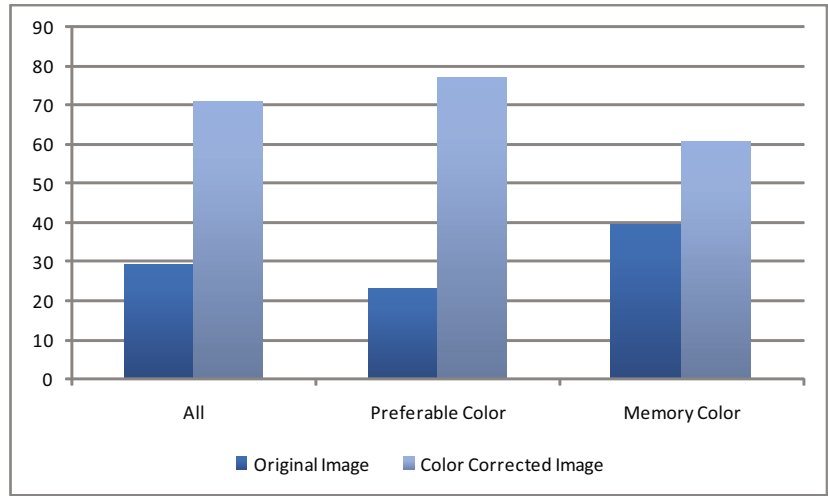

Fig. 6. Acceptability percentage for original image vs. color corrected image. Statistics for models, using preferable color and memory color.

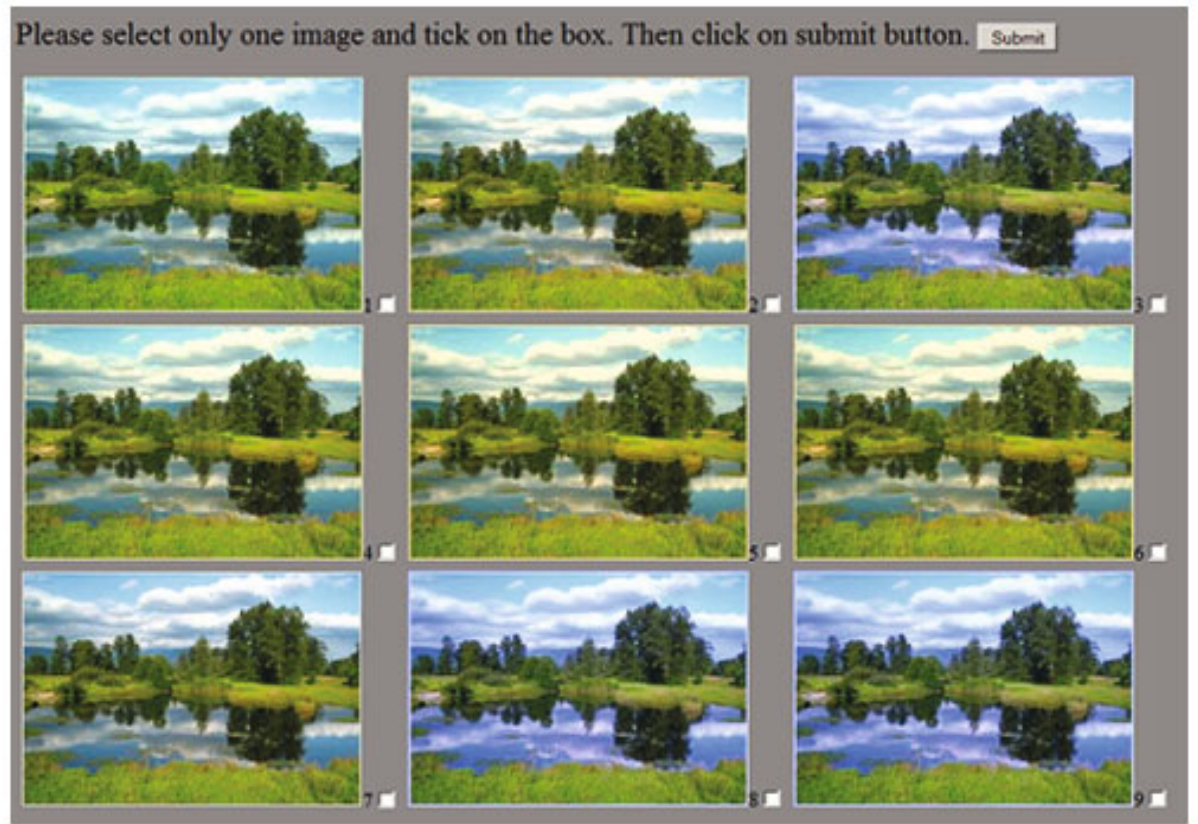

Fig. 7. Sample of online visual experiment. The 8 color corrected images are shown together with the original one. The original image is number 1. 


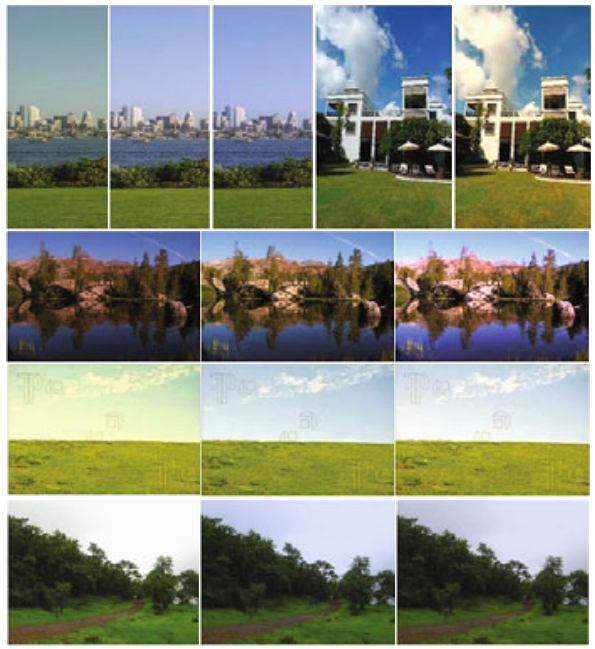

Fig. 8. Differences between some of the proposed color corrected images

Table 1. $\mathrm{L}^{*} \mathrm{a} \mathrm{b}^{*}$ values of the memory colors without context information

\begin{tabular}{|c|c|c|c|c|c|c|c|c|c|c|c|c|c|}
\hline \multirow{2}{*}{ Lighting } & \multirow{2}{*}{ Object } & \multicolumn{3}{|c|}{ Mean } & \multicolumn{3}{|c|}{ Variance } & \multicolumn{3}{|c|}{ Max } & \multicolumn{3}{|c|}{ Min } \\
\hline & & $L^{*}$ & $a^{*}$ & $b^{*}$ & $L^{*}$ & $a^{*}$ & $b^{*}$ & $\mathrm{~L}^{*}$ & $\mathrm{a}^{*}$ & $b^{*}$ & $\mathrm{~L}^{*}$ & $a^{*}$ & $b^{*}$ \\
\hline \multirow{3}{*}{ Dark } & rass & 73.93 & -31.21 & 45.05 & 3.03 & $\overline{230.81}$ & 208.03 & 5.74 & -6.6 & 59.89 & 71.6 & -56.39 & 23.51 \\
\hline & & 63.27 & -17.63 & -8.73 & 4.91 & 47.13 & 96.81 & 65.38 & -10.11 & 2. & 59.64 & 31.94 & -23.14 \\
\hline & $\mathrm{S}$ & 67.22 & 0.18 & 16.43 & 0.02 & 0.04 & 0.73 & 67.64 & 0.53 & 18.94 & 67.1 & -0.02 & 15.62 \\
\hline \multirow{3}{*}{ Dim } & Gras & 73.98 & -32.57 & 44.6 & 3.33 & 135.6 & 233.73 & 76.08 & -10.25 & 60.22 & 71.6 & -46.52 & 22.94 \\
\hline & & 63.5 & -18.36 & -8.08 & 3.09 & 60.08 & 59.86 & 65.2 & -9.55 & 0.9434 & 59.3 & $\mid-29.6$ & -24.25 \\
\hline & Snow & 67.27 & -0.24 & 16.7 & 0.02 & 0.05 & 0.64 & 67.33 & 0.5 & 17.09 & 66.93 & -0.19 & 14.75 \\
\hline \multirow{3}{*}{ Bright } & Grass & 74.05 & -30 & $\overline{47.23}$ & 4.7 & 248.1 & 297.34 & 76.17 & -5.7 & 62.24 & 70.18 & -50.6 & 21.47 \\
\hline & Sky & 63.84 & -22.16 & -7.86 & 1.55 & 42.72 & 22.22 & 65.55 & -12.62 & -3.79 & 61.19 & -34.31 & -16.53 \\
\hline & Snow & 67.61 & -1.9 & 17.68 & 0.04 & 0.06 & 1.19 & 68.09 & -1.67 & 20.53 & 67.47 & -2.18 & 17.01 \\
\hline
\end{tabular}

Table 2. $\mathrm{L}^{*} \mathrm{a}^{*} \mathrm{~b}^{*}$ values of the prefereable memory colors using images

\begin{tabular}{|c|c|c|c|c|c|c|c|c|c|c|c|c|c|}
\hline \multirow{2}{*}{ Lighting } & \multirow{2}{*}{ Object } & \multicolumn{3}{|c|}{ Mean } & \multicolumn{3}{|c|}{ Variance } & \multicolumn{3}{|c|}{ Max } & \multicolumn{3}{|c|}{ Min } \\
\hline & & $\mathrm{L}^{*}$ & $a^{*}$ & $b^{*}$ & $\mathrm{~L}^{*}$ & $a^{*}$ & $b^{*}$ & $\mathrm{~L}^{*}$ & $a^{*}$ & $\mathrm{~b}^{*}$ & $\mathrm{~L}^{*}$ & $a^{*}$ & $b^{*}$ \\
\hline \multirow{3}{*}{ Dark } & ass & 73.2 & 30.63 & 40.08 & 5.68 & 229 & 229 & 6.17 & -1.93 & 59.89 & .37 & -58.85 & 3.23 \\
\hline & & 64.84 & ]$^{-1}$ & 0.24 & 2.05 & 53.76 & 69.83 & 66.49 & -1.5 & 11.19 & .56 & -21 & -15. \\
\hline & $\mathrm{S}$ & 67.17 & 5.33 & 20.19 & -0.03 & 323.31 & 195.09 & 67.64 & 62.42 & 64.43 & 66.93 & -0.29 & 14.75 \\
\hline \multirow{3}{*}{ Dim } & Grass & 73.4 & 26.94 & 43.08 & 4.26 & 183.57 & 159.43 & 76.12 & \begin{tabular}{|l|}
-6.32 \\
\end{tabular} & 60 & 69.36 & -59.21 & 18.28 \\
\hline & Sky & 63.92 & 1.81 & -4.21 & 3.35 & 33.35 & 81.36 & 66.51 & 1.27 & 11.88 & 59.31 & -24.28 & -24.25 \\
\hline & Snow & 67.31 & -2.91 & 15.82 & 0.03 & 116.77 & 16.4 & 67.75 & 0.5 & 19.63 & 67.09 & -37.22 & 3.35 \\
\hline \multirow{3}{*}{ Bright } & Grass & 73.97 & -27.03 & 47.36 & 3.26 & 111.47 & $\overline{174.54}$ & 76.17 & -14.76 & 63.49 & 70.91 & -52.41 & 25.26 \\
\hline & Sky & 64.21 & -17.58 & $\mid-4.7$ & 2.06 & 55.76 & 63.14 & 67.23 & -6.2 & 13.74 & 62.18 & -31.16 & -14.8 \\
\hline & Snow & 67.54 & -2.01 & 17.8 & 0.02 & 0.06 & 0.34 & 67.72 & -1.67 & 18.23 & 67.47 & -2.18 & 17.01 \\
\hline
\end{tabular}




\section{Conclusion}

A novel color correction process based on a weighted Von Kries model and a new approach to acquire memory color data by exploiting the advantages of multispectral imaging is presented in this paper. Proposed color correction process utilizes multiple memory color objects present in the image to generate pleasing color corrections. The results reveal that $70 \%$ of the users preferred the color corrected images. It must be noted that color preferences are very subjective, hence it becomes difficult to propose one type of correction that will suit all observers. Nonetheless, the proposed model is capable of providing pleasant color corrections which suits the majority of the observers.

\section{References}

1. Breiman, L.: Random forests. Machine Learning 45, 5-32 (2001), 10.1023/A:1010933404324

2. Fairchild, M.D.: Color Appearance Models, 2nd edn. Wiley-IS\&T, UK (2005)

3. Fredembach, C., Estrada, F., Süsstrunk, S.: Memory colour segmentation and classification using class-specific eigenregions. Journal of the Society for Information Display 17, 921-931 (2009)

4. Gage, J.: Color and Culture: Practice and Meaning from Antiquity to Abstraction. Thames \& Hudson, UK (1993)

5. Hanbury, A.: A 3D-polar coordinate colour representation well adapted to image analysis. In: Bigun, J., Gustavsson, T. (eds.) SCIA 2003. LNCS, vol. 2749, pp. 804-811. Springer, Heidelberg (2003)

6. Ishihara, S.: Tests for colour blindness (1917)

7. Jobson, D.J., Rahman, Z., Woodell, G.A.: Statistics of visual representation, vol. 4736, pp. 25-35. SPIE, Bellingham (2002)

8. Kaiqi, H., Zhenyang, W., Qiao, W.: Image enhancement based on the statistics of visual representation. Image and Vision Computing 23(1), 51-57 (2005)

9. Lopez-Moreno, J., Hadap, S., Reinhard, E., Gutierrez, D.: Compositing images through light source detection. Computers \& Graphics 34(6), 698-707 (2010); Graphics for Serious Games; Computer Graphics in Spain: a Selection of Papers from CEIG 2009; Selected Papers from the SIGGRAPH Asia Education Program

10. Lu, A., Xu, G., Jin, H., Mo, L., Zhang, J., Zhang, J.X.: Electrophysiological evidence for effects of color knowledge in object recognition. Neuroscience Letters 469(3), 405-410 (2010)

11. Rahtu, E., Nikkanen, J., Kannala, J., Lepistö, L., Heikkilä, J.: Applying visual object categorization and memory colors for automatic color constancy. In: Foggia, P., Sansone, C., Vento, M. (eds.) ICIAP 2009. LNCS, vol. 5716, pp. 873-882. Springer, Heidelberg (2009)

12. Roberson, D., Davidoff, J., Davies, I.R.L., Shapiro, L.R.: Color categories: Evidence for the cultural relativity hypothesis. Cognitive Psychology 50, 378-411 (2005)

13. Stepan, O., Jiri, M., Ondrej, C.: On the interaction between object recognition and colour constancy, vol. 45, pp. 5-32. IEEE Computer Society, Los Alamitos (2003)

14. van de Weijer, J., Schmid, C., Verbeek, J.: Using high-level visual information for color constancy. In: IEEE 11th International Conference on Computer Vision, ICCV 2007, pp. $1-8$ (2007)

15. Zhang, H., Quan, S.: Memory color assisted illuminant estimation through pixel clustering, vol. 7537, p. 75370J. SPIE, Bellingham (2010) 